\title{
Glycoalkaloids as medicinal agents from callus and regenerated plants of Solanum nigrum var. judiacium
}

Hanan Abd Al-Hay Al-Ashaal*

National Research Centre, Dokki, Egypt

*Corresponding author e-mail: hanan_alashaal@yahoo.com.

\begin{abstract}
The main target of this study is production of glycoalkaloids from in vitro cultures of Solanum nigrum var. judiacium Besser. Further, to evaluate their therapeutic effects. S. nigrum var. judiacium leaves were implanted in solid MS media containing growth regulators for in vitro study. HPLC analyses were applied for qualitative and quantitative determination of glycoalkaloids. Cytotoxic effects against human carcinoma cell lines were evaluated. In addition, antiviral, antioxidant, antiinflammatory and antiparasitic activities of glycoalkaloids were estimated. HPLC data indicated the success of in vitro solasodine and solanidine glycosides production. Solasonine represented the highest concentration. Biological assays showed that obtained glycoalkaloids exhibited cytotoxic activity against human carcinoma cell lines that may be attributed to free radical scavenging activity (69.98\%). Strong antiherps performance was recorded (94\%). In addition, the glycoalkaloids showed in vitro schistomicidal $\left(I C_{50} 76.4 \mathrm{ppm}\right)$ and fasciolicidal $\left(I C_{50} 76.6 \mathrm{ppm}\right)$ activities. In vivo anti-inflammatory assay revealed potent activity against carrageenan induced edema. Glycoalkaloids were formed 2-5 folds that of intact plant pointed to the efficiency of the cultures. The present findings referred to the pronounced biological performance of the in vitro produced glycoalkaloids including antiviral, cytotoxic anti-inflammatory and antiparasitic activities. Botanical derived medication from S. nigrum var. judaicum could be accomplished guided with the present data.
\end{abstract}

Key words: Solanum nigrum var. judiacium, tissue culture, biological activity

\section{Introduction}

Medicinal plants have been taking place in modern remedy as raw materials for some important medicines (Gheewalaa et al., 2013). Solanaceae, an economically and medicinally important family is characteristic of having alkaloid accumulation (Kumar et al., 2009). Solanum nigrum var. judiacium Besser belongs to genus Solanum (Night shades), family solanaceae and accumulates steroidal alkaloids called glycoalkaloids.

These glycoalkaloids constitute a paramount class of phytochemicals having distinct medicinal applications including antiviral, anticancer and antiparasitic therapeutic agents.
Solanine, an important solanidine glycoalkaloid found in some Solanum species, is reported to have antimicrobial, antidiabetic, antiallergic and anticarcenogenic effects based on apoptotic efficacy (Kumar et al., 2009; Williams, 2013). In previous work, it was reported that $S$. tuberosum glycoalkaloids; a-Chaconine, a-Solanine and Solanidine exhibited noticeable cytotoxic and antiviral activities. The author demonstrated likewise, their antiparasitic performance as antischistomiasis and antifasciolosis (Al-Ashaal, 2010). Solasonine and solamargine glycoalkaloids were reported to have anticarcenogenic effect against different carcinoma cell lines including glioblastoma multiform, liver, basal cell and 
squamous, bladder, and colon rectal cell lines (Tek, 2006). In addition, solamargine and crude Solanum palinacanthum ethanolic extract were adduced to have trypanocidal effectiveness against the causative parasite of Chagas' disease (Moreira et al., 2013). Solasonine and solamargine glycoalkaloids as well, are economically important because they are structurally similar to steroidal hormones and have been utilized as a valuable source for medicines, such as contraceptives and steroidal anti-inflammatory medicaments (Tiossi et al., 2012).

Regrettably, there is limited use of herbal drugs because of several reasons such as poor availability and traditional cultivation technology. Moreover, plant secondary metabolites concentration in a given plant often varies during 24-hour period (Tek, 2006). Several factors during growth, harvesting and post-harvest treatment, and maturity can affect glycoalkaloid accumulation (Tek, 2006). Genotype has a major effect on glycoalkaloid levels. It was proposed that any environmental factor which causes a stress in a plant of the Solanum species can alter glycoalkaloid content (TeK, 2006). For the medicinal and pharmaceutical importance of glycoalkaloids, and their poor availability in many cases, attempts for in vitro production have been documented. The production of alkaloids in plant tissue culture by some solanaceous species including solanum have been reported in different studies (Lindesy\& Yeoman, 1983; Misawa, 1994; El-Ashaalal., 1999; Moreeira et al., 2010; Al-Ashaal et al., 2013).

Due to their significance medical value, the present study focuses on S. nigrum var. judiacium as a source of glycoalkaloids. Modulating culture conditions to produce considerable concentrations of glycoalkaloids from in vitro cultures in good supply for pharmaceutical industry is basic objective of the present study. The other interesting target is to estimate the bioactivity of the in vitro produced glycoalkaloids with the intent of producing effective and available therapy from in vitro cultures. Consequently, establish the in vitro guidelines for handling the medicinal important glycoalkaloids from S. nigrum var. judiacium for pharmaceutical applications.

\section{Material and Methods}

Experimental tools. Solanum nigrum var. judiacium leaves were obtained from the farm of medicinal plants, Faculty of Pharmacy, Cairo University, Egypt. A voucher specimen of the regenerated plants was identified by Prof. Dr. Mounir Abd El-Ghany and deposited at Cairo University Herbarium (CAl) with registration number CAl 343216.

Standard solasonine and solamargine, Sigma-Aldrich Co., St Louis, were kindly provided by Dr. Ashgan Zaki Prof. of Pharmacognosy Faculty of Pharmacy Cairo University. Solasodine ( $\geq 99 \%$ ), Sigma Co., a-solanine, ( $\geq$ N97\%) Roth Co.; Cisplatin injectable grade (98-102\%) from Merck Co.; Doxorubicin injectable grade (98-102\%) from Pharmacia Co.; Taxol injectable grade (98-102\%) from Mayne Pharm. Co.; DPPH• (2, 2-Diphenyl-1-picrylhydrazyl - 97\%) from Sigma Co.; Vitamin C (N99.5\%) from Fluka Co.; Acyclovir (N95\%) from Sigma Co.; Braziquantel (97.5-102\%) from Alexandria Co. for pharmaceuticals.; Carrageenan ( $\geq 98 \%$ ) Sigma Co.; Indomethacin, El- Kahera Pharmaceutical Industry Co., Egypt. Media components and phytohormones for regeneration and in vitro glycoalkaloids production were tissue culture grade. Solvents for analysis were HPLC grade.

HPLC; Hewlett Packard (Wilmington, North Carolina, United State), series 1050, UV detector, wave length $210 \mathrm{~nm}$, column C18 5 $\mu \mathrm{m}, 0.4 \times 25 \mathrm{~cm}, \mathrm{FR} .1 \mathrm{~mL} / \mathrm{min}$. Pressure 6 bar, temp. $40^{\circ} \mathrm{C}$.

ESR; Bruker, Elexys, (Silberstreifen, Rheinstetten, Germany),

$X$-band modulation frequency 500 $\mathrm{MHz}$. The sample inserted via quartz liquid flat cell, average scans 1, average sampling time (s) 0.04096, state of aggregation C, field Mod. Amplitude 0.0002, field Mod. Frequency (Hz) 100,000 microwave frequency $(\mathrm{Hz}) 9.77568 \mathrm{e}+09$, microwave power (w) 0.00202637, receiver gain 65, receiver harmonic.

Light microscope; Olympus, Saitama, Japan, Eye piece: 25X, Oil objective: 100X.

Plethysmometer 7150, (UGO, Basil, Italy).

In vitro cultures and qualitative \& quantitative analyses of glycoalkaloids. Solanum nigrum var. 
judiacium leaves were sterilized with Clorox (10\% $\mathrm{v} / \mathrm{v}$ sodium hypochlorite solution) for $20 \mathrm{~min}$, immersed in $70 \%$ ethyl alcohol for 15 seconds, and then rinsed with sterile distilled water twice, in laminar airflow cabinet. The leaves were sliced and aseptically cultured in glass jars containing MS media (Murashige \& Skoog, 1962) supplemented with growth regulators including auxins and cytokinins as BA, IAA, NAA and 2, 4-D at different ratios. The cultures maintained at $26^{\circ} \mathrm{C}$ and $16 / 8$ light photoperiod. Subcultures of callus cultures on fresh media were performed every 4-6 weeks. Regenerated shoots were subcultured on MS media supplemented with hormones for two subcultures then resubculture in basal media to allow rooting. Acclimatization of regenerated plants was carried according to El-Ashaal et al. (1999).

HPTLC silica plates were used for qualitative identification of in vitro glycoalkaloids. Fifty mg of dried calli were extracted with $96 \%$ methanol twice $(2 \times 100 \mathrm{~mL})$. The extract was concentrated under vacuum and the residue was co-chromatographed against standards. Eluting system for the glycoalkaloids was chloroform- methanol-1\% ammonia 2:2:1 v/v (the organic layer). While that for aglycone was benzene- methanol 4:1 v/v.

HPLC quantitative analysis was carried using isocratic mode. Plant materials from callus, regenerated shoots, fruits of the acclimatized in vitro plants and mother field Solanum leaves were dried at a temperature $45^{\circ} \mathrm{C}$. Ten $\mathrm{mg}$ of the dried materials was crushed, extracted with $25 \mathrm{~mL}$ methanol $(96 \%)$ in water bath at $50^{\circ} \mathrm{C}$ for $3 \mathrm{~h}$, and subsequent homogenized in methanol using ultra-turrax three times each for $5 \mathrm{~min}$. (3 $\times 15 \mathrm{~mL}$ ), the combined extracts were collected and concentrated under reduced pressure and temperature. The residue of the test materials in addition to standard glycoalkaloids were dissolved in methanol $(1: 1 \mathrm{~W} / \mathrm{v})$ and filtered through $0.45 \mu$ Millipore filters then analyzed by HPLC in triplicates $10.25-1 \mu L$, injection volume depending on the concentration of each sample) using $40 \%$ methanol as mobile phase, wave length, $210 \mathrm{~nm}$, flow rate $1 \mathrm{~mL} /$ min. Temperature $40{ }^{\circ} \mathrm{C}$. Authentics standard curves were plotted. The concentrations of the glycoalkaloids were determined established on the calculation of percentage peak areas of the samples comparing with that of standards. The regression process was carried out and the following functions for different standards were drawn out:

For solasonine: $Y=45.55 X-44.25 \quad r=0.972$

For a-solanine: $Y=5.281 X+2.1485 \quad r=0.994$

For solamargine: $Y=102.4 X-41.83 \quad r=0.967$

For solasodine: $Y=129.5 X-230 \quad r=0.999$

Concerning isolation of in vitro produced glycoalkaloids; calli were dried at a temperature $45{ }^{\circ} \mathrm{C}$, mashed in coarse powder and then, extracted with $5 \%$ acetic acid twice. The combined extracts were filtered and the filtrate was concentrated under vacuum pressure. The concentrate was treated with ammonium hydroxide till pH 10 then pure glycoalkaloids were precipitated upon cooling.

\section{Medicinal investigation}

Cytotoxic potential against human carcinoma cell lines. Cytotoxic activity of in vitro produced glycoalkaloids was performed against different strains of breast (MCF7-HTB-22), lung (H460), liver (Hepg2-ATCCHB-8065), and brain (U251NIBIO IF050288) human carcinoma cell lines at the National Cancer Institute, Egypt, according to the reported method of Skehan et al. (1990). While, the tested material was examined for cytotoxicity against lymphoblastic leukemia (1301) at the National Research Center, Egypt, following the described technique (Hansen et al., 1989). $I C_{50}$ values $(\mathrm{\mu g} / \mathrm{mL})$ were determined comparing with standard cytotoxic drugs. Negative control groups were also performed. Cytotoxicity against the selected cell lines was examined in triplicates.

Antioxidant capability. Free radical scavenging capacity of the isolated glycoalkaloids was measured by spectrophotometric method using electron spin resonance technique (ESR) (AlAshaal, 2010) in comparison with a -solanine and vitamin $C$ as positive control against free radical diphenyl picryl hydrazyl (DPPH*). $1 \mathrm{~mL}$ of 10-3 MDPPH was added to $1 \mathrm{mg}$ of standard a -solanine, vitamin C or $1 \mathrm{mg}$ of the isolated glycoalkaloids. Measurements were taken after 
5 minutes. Antioxidant capacity was function of reduction of integrated areas of $D P P H^{*}$.

$\%$ of antioxidant capacity $=A_{0}-A_{1} / A_{0} \times 100$

$\mathrm{A}_{0}=$ Area of $\mathrm{DPPH}^{\cdot}$

$\mathrm{A}_{1}=$ Area of the tested sample+ $\mathrm{DPPH}^{\cdot}$

Antiviral examination. Preliminary antiviral investigation was performed against Herpes simplex virus type 1 (HSV-1) as a model of DNA virus. The virus was isolated and proliferated in the virology Laboratory of the Department of Water Pollution, National Research Center (Egypt). African green monkey cells (Vero) were used as virus host. The non toxic concentration range of the tested sample (in 10\% dimethylsulphoxide; DMSO) to Vero cells was determined and the virucidal efficacy was measured (Papageorgiou et al., 2000). The percentage of virus plaques reduction was calculated and taken as measurement of the antiviral activity. Acyclovir was used as positive antiviral control drug and untreated virus and cells in 10\% DMSO as negative control.

Antiparasitic prospect. The vermicidal activity of the isolated glycoalkaloids was tested against Schistosoma mansoni and Fasciola gigantica worms. Schistosoma worms were provided from Theodor Belharz Research Institute, Egypt. Fasciola worms were derived from the infected buffalo's livers at Cairo abattoir, Egypt. The experiments were performed in triplicates using RPMI media provided with antibiotics (Hassanain, 1998). Activity of the isolated glycoalkaloids was tested at concentrations (40-200) ppm. Braziquantel was used as positive control drug at concentration of $100 \mathrm{ppm}$. Negative control without glycoalkaloids was also performed. Observation of mortality was achieved using inverted microscope, after 24, 48, 72 and $96 \mathrm{~h}$ for S. mansoni and after $24 \mathrm{~h}$ for F. gigantica as the worms are susceptible, and may not remain survival after $24 \mathrm{~h}$. Analysis was performed using probit program and $I C_{50}$ determined at $95 \%$ confidence limit (Finney, 1971).

Anti-inflammatory potency. In vivo antiinflammatory activity was performed using carrageenan induced oedema method (Winter 1962). Male albino rats weighing (70- $80 \mathrm{~g}$ ) were obtained from the National Research Center animal laboratory (Giza, Egypt). The rats maintained on water and stock commercial pellet diet ad libitum. Handling, procedures were carried out according to the approved institutional and international ethical guidelines for laboratory animals' use and care. Animals were divided into 4 groups each of 5 rats. First control negative group received $0.1 \mathrm{~mL}$ saline. second group each rat received in the left hind paw indomethacin $(20 \mathrm{mg} / \mathrm{kg}$ i.p) $\mathrm{th}$ before carrageenan administration. Third and fourth groups each rat received glycoalkaloids in the left hind paw at dose equivalent to $1 / 4$ or $1 / 2$ $L D_{50}$ (Tek, 2006) (8 or $16 \mathrm{mg} / \mathrm{kg}$ i.p) in before carrageenan administration. All animals except Ist group were given a sub-plantar injection of $0.1 \mathrm{ml}$ of $1 \%$ carrageenan solution in saline in the right hind paw. The paw volume of each rat was measured before carrageenan injection and then hourly at intervals up to four times with Plethysmometer. The oedema rate and inhibition rate of each group were calculated.

Oedema inhibition percentage was calculated according to the following equation: Inhibition $\%=$ Inhibition rate - Rate of edema/ Rate of oedema x 100.

Statistical analysis was carried using one way ANOVA Post Hoc test.

Dependent variable: VAR 00002, LSD at $95 \%$ confidence interval.

\section{Results and Discussion}

In vitro cultures and glycoalkaloids estimation. Murashige \& Skoog (MS) media supplemented with BA and 2, 4-D in a ratio of 1: 4 was the best medium for callus initiation while the best medium for shoot induction was that contained BA and NAA in a ratio of 1:1 with flowers in some cultures (Figures. 1-3).

Rooting was achieved successfully in MS basal media. Fruits were well developed in the acclimatized plants. Media contained 2, 4 -D did not show any differentiation. This is in close agreement with formerly reports elucidated that BA and NAA induced shoots in S.nigrum var. judaicum cultures but at ratio (0.5: 1) (El-Ashaal, 1999). AS well as, it was reported that roots were developed in MS media contained IAA and BA or NAA and BA in different rates in S. torvum cultures (Moreeira et al., 2010). 

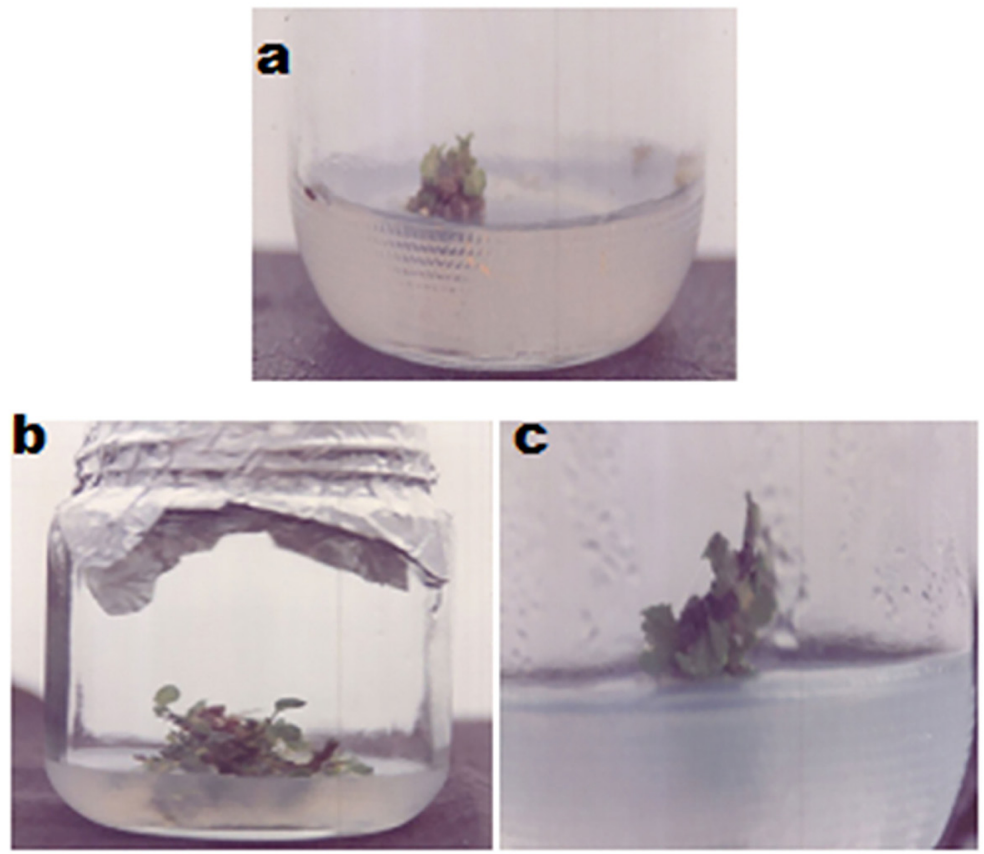

Figure 1. Primordial of regeneration cultures from leaf explants of $S$. nigrum var. judaicum after 1 week (a), development of regeneration after 2 weeks (b), regenerated calli after 4 weeks (c).

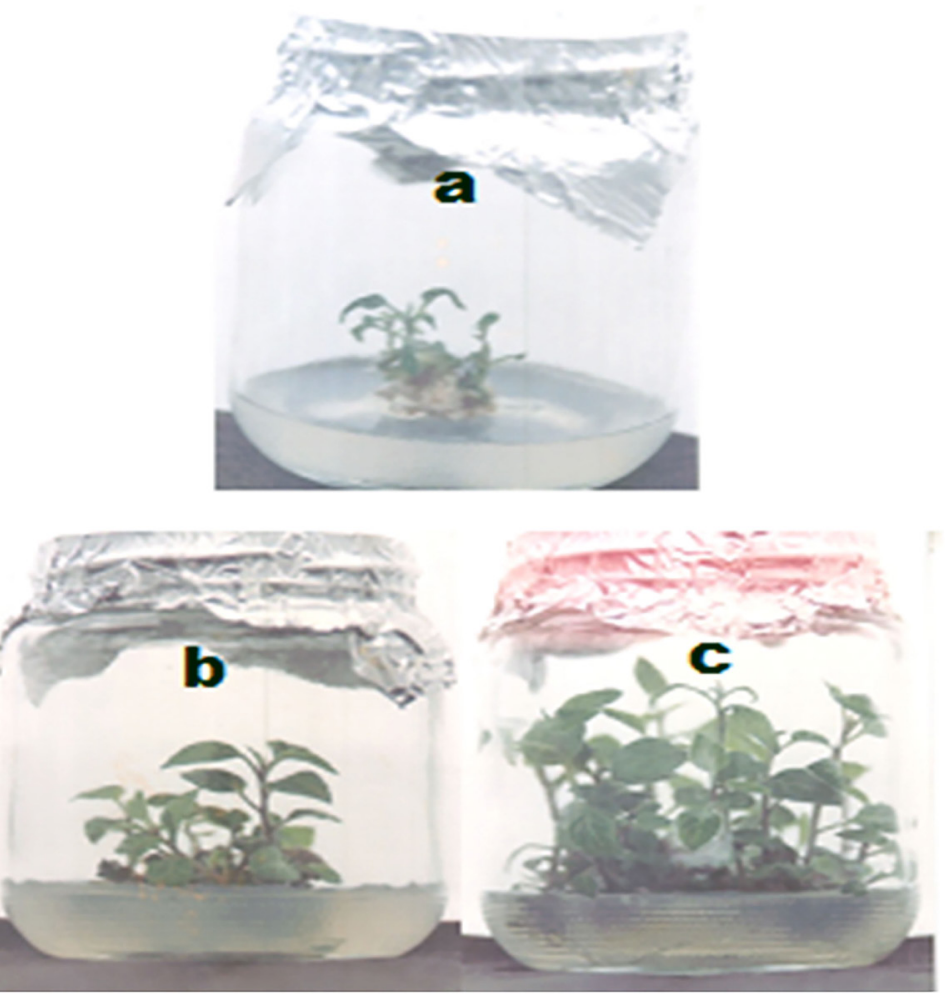

Figure 2. Shoots initiation after 4 weeks (a), 6 weeks (b) and 8 weeks (c) of S. nigrum var. judiacium. 


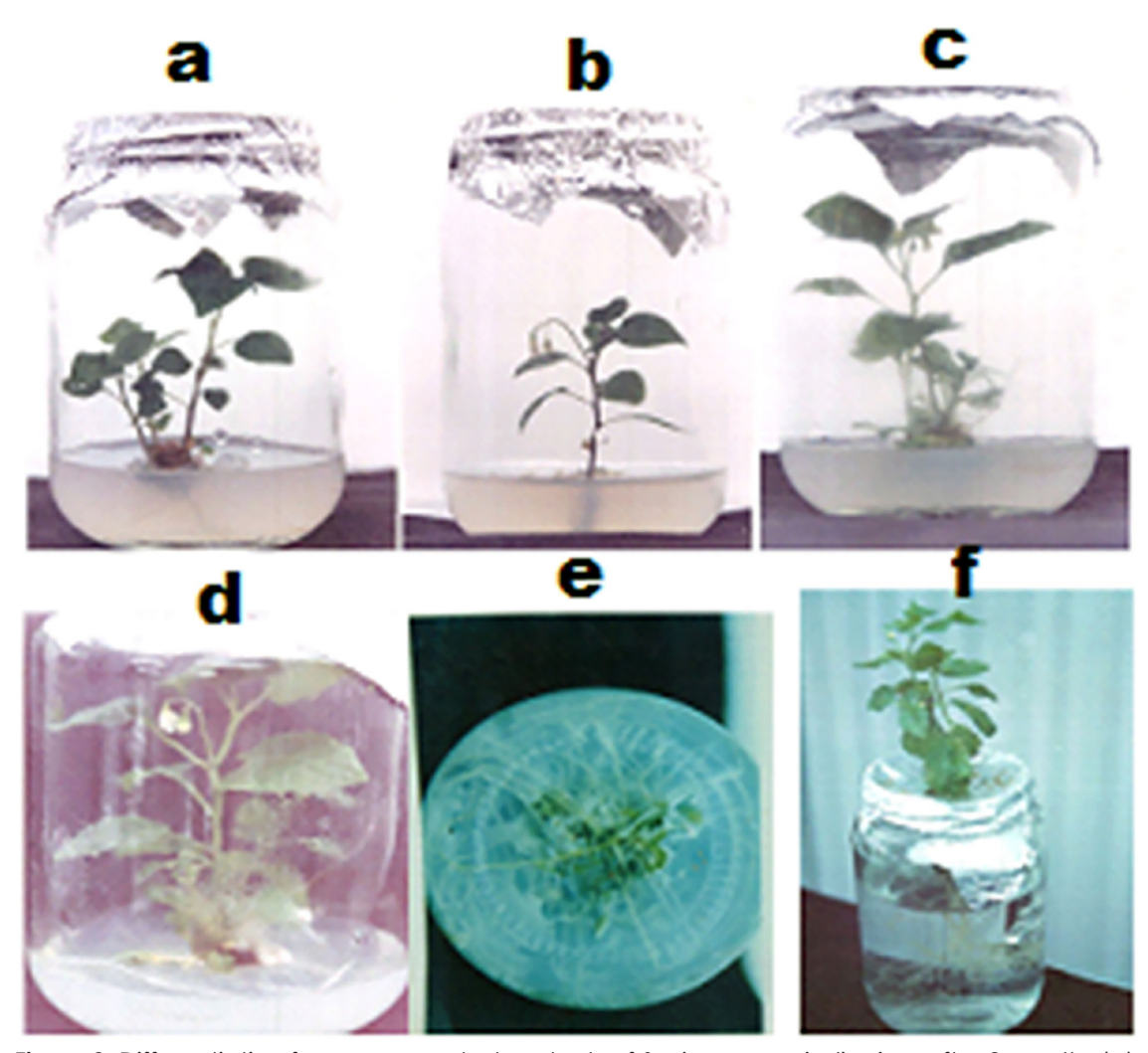

Figure 3. Differentiation from regenerated explants of $S$. nigrum var. judiacium after 2 months (a), flowering of subcultured differentiated explants after one subculture (b), two subcultures (c), three subcultures (d), rooting after 3 months (e) and adapted plant (f).
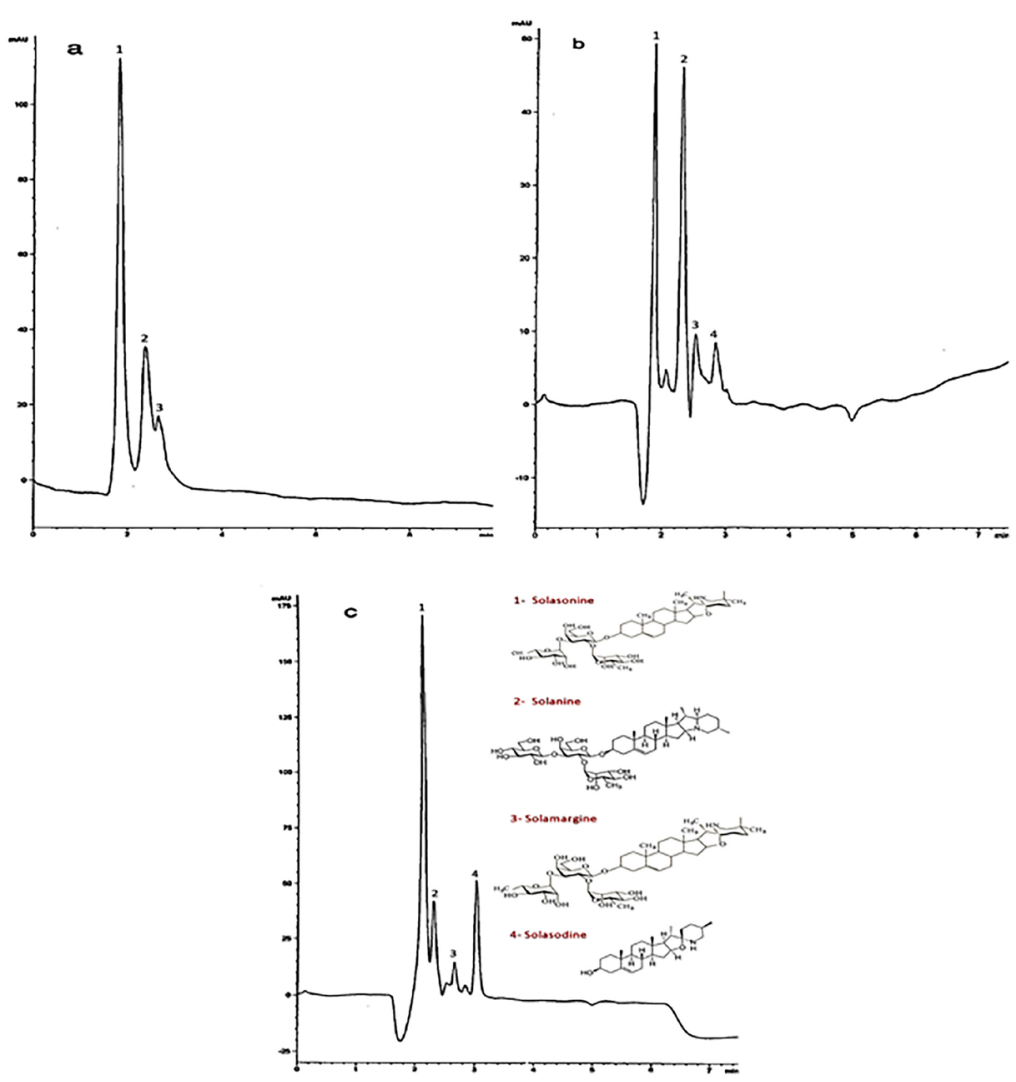

Figure 4. HPLC of in vitro glycoalkaloids of $S$. nigrum var. judiacium: $a$; Field plant derived glycoalkaloids, b; regenerated plant fruits derived glycoalkaloids, c; In vitro callus derived glycoalkaloids 
HPTLC and HPLC analysis showed that calli, differentiated shoots and ripe fruits of acclimatized in vitro plants produced solasonine, solanine and solamargine glycoalkaloids in addition to solasodine (Figure 4).

Crystallization of callus methanolic extract showed prisms of solasonine, yellow needles of solamargine and slender transparent needles of solanine (Figure 5).

Complete adapted cycle was represented starting with tissue culture stages and end with extracting glycoalkaloid crystals (Figure 6). Data of HPLC analyses (Table 1) illustrated that solasonine had the highest concentration. In accordance with the present data, solasonine was found to constitute the major glycoalkaloids in S. nigrum fruits (Gheewalaa et al., 2013).

The present results likewise, indicated that glycoalkaloids were produced in cultures in much higher concentrations comparing with field plant derived leaves. The increment reached 3.03, 2.48 and 4.10 folds for callus cultures. While, the increments were 2.56, 2.88 and 4.74 folds for regenerated shoots. Finally, for fruits the increments were 2.51, 4.05 and 5.17 folds for solasonine, solanine and solamargine respectively. It is important to know that the concentrations of glycoalkaloids in Solanum plants varied dramatically and sometimes becomes nil (Bhatnagar et al., 2004). In spite that certain Solanum species contain high concentrations of glycoalkaloids (concentrations of some Solanum species were between $110 \mathrm{mg}$ and $890 \mathrm{mg} / 100 \mathrm{~g} \mathrm{FW}$ ) (Väänäne, 2007) yet, the concentration of glycoalkaloids could be altered due to environmental factors and stress (Tek, 2006).

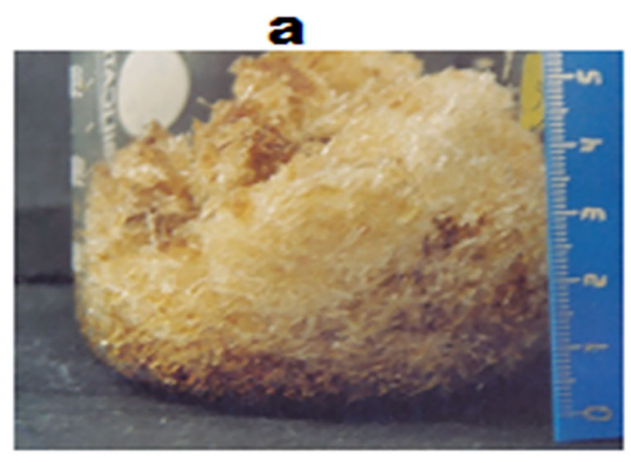

b

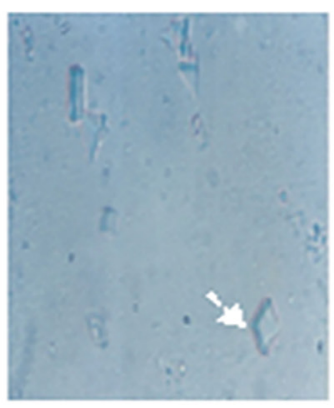

Figure 5. Crystals of in vitro glycoalkaloids of S. nigrum var. judiacium: a ; Collective crystals (Photo), b-Prisms of solasonine (25x100), c ;Transparent needles of solanine (25x100), d ;Long yellow needles of solamargine (25x100) e; Transparent slender needles of solanine

Table 1. In vitro glycoalkaloid content of S.nigrum var. judiacium. (\% dry wt.).

\begin{tabular}{lllll}
\hline R f & Rsh & Ca. & Ml & Glyc. \\
\hline 35.14 & 35.84 & 42.35 & 14.00 & Solasonine \\
29.42 & 20.91 & 18.00 & 7.26 & Solanine \\
8.90 & 8.15 & 7.06 & 1.72 & Solamargine \\
11.47 & 8.75 & 9.45 & - & Solasodine \\
\hline Glyc.: glycoalkaloid; Ml : mother derived leaves; Ca: callus; Rsh: regenerated shoots; Rf: regenerated plant fruits
\end{tabular}


Convenient with the present results, In vitro alkaloids production has been documented (Misawa, 1994; El-Ashaal et al., 1999; Moreeira et al., 2010). The current data showed that 2 , 4-D enhanced glycoalkaloids production which is consistence with previous finding (Lindesy\& Yeoman, 1983) illustrated that 2, 4-D enhanced alkaloid production in Datura innoxia cultures. Although, no shoots were developed in media contained 2, 4-D in the present study, other researchers reported regeneration of $S$. nigrum cultures on MS media containing $1.0 \mathrm{mg} / \mathrm{L} 2$, 4-dichlorophenoxyacetic acid, under dark condition (XU et al., 2014). The current work showed that auxins and cytokinins were essential for glycoalkaloids production. On contrast, it was found that $S$. torvum plants grown in MS media produced more solasodine than those grown in MS with auxins and cytokinin added (Moreeira et al., 2010). The high glycoalkaloids accumulation in the present work is compatible with the reported high yield of in vitro anthraquinones at concentration of $18 \%$ on dry weight bases in cultures of Morinda citrifolia comparing with $0.3 \%$ of mother plant that represents 60 folds (Misawa, 1994). Higher yield of glycoalkaloids from $S$. tuberosum cultures comparing with field tubers was also reported (Al-Ashaal, 2010). The author accused the high yield to good selection of the original strain, combination of growth regulators and adjustment of cultured conditions which may also be the case in the current study. The present work enables high glycoalkaloids yield from $S$. nigrum var. judiacium cultures. This achievement could be helpful for providing these important phytochemicals for pharmaceutical drugs in stabilized manner.
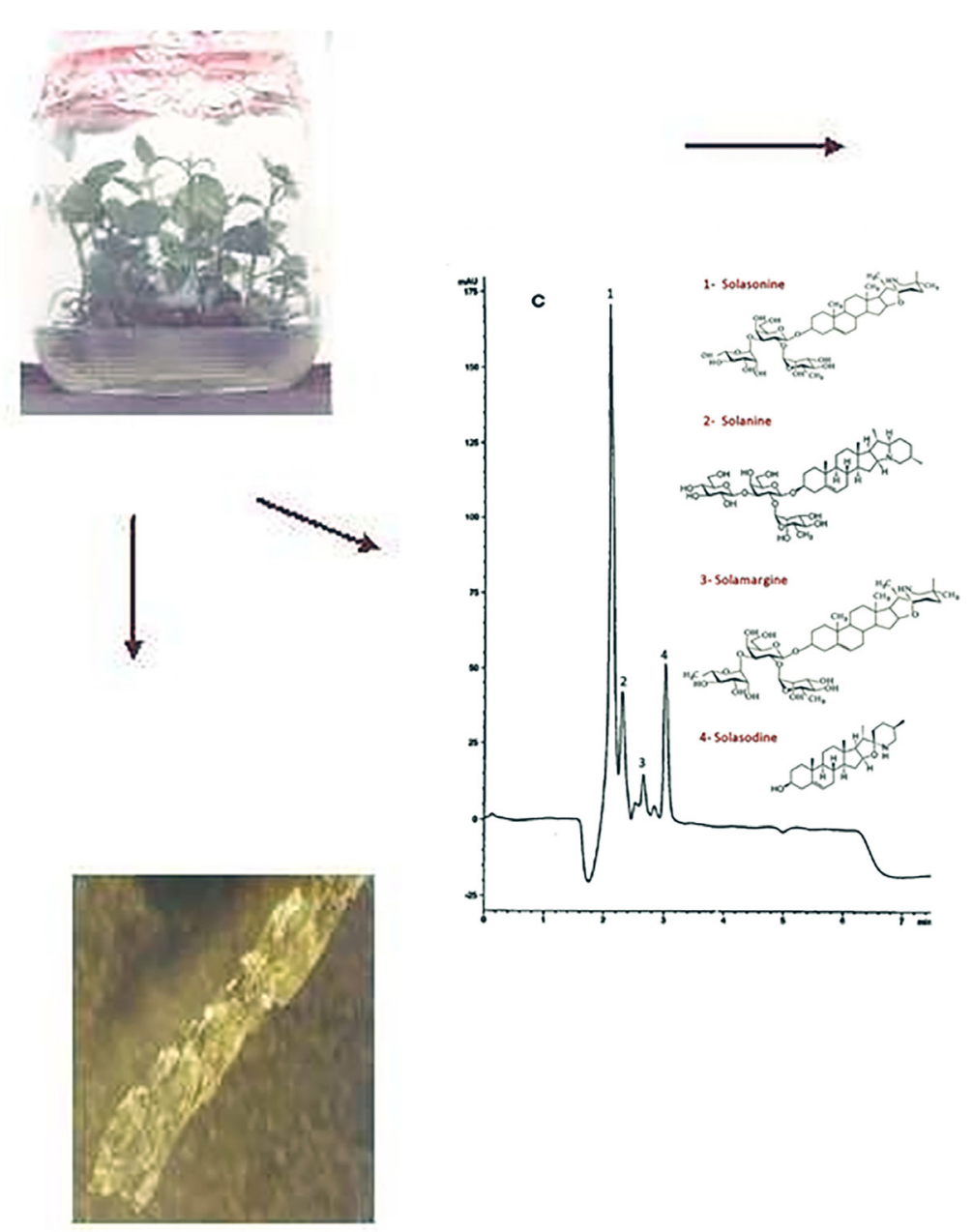

Figure 6. Illustration of whole in vitro process beginning with tissue culture cycle and end with glycoalkaloid crystals.
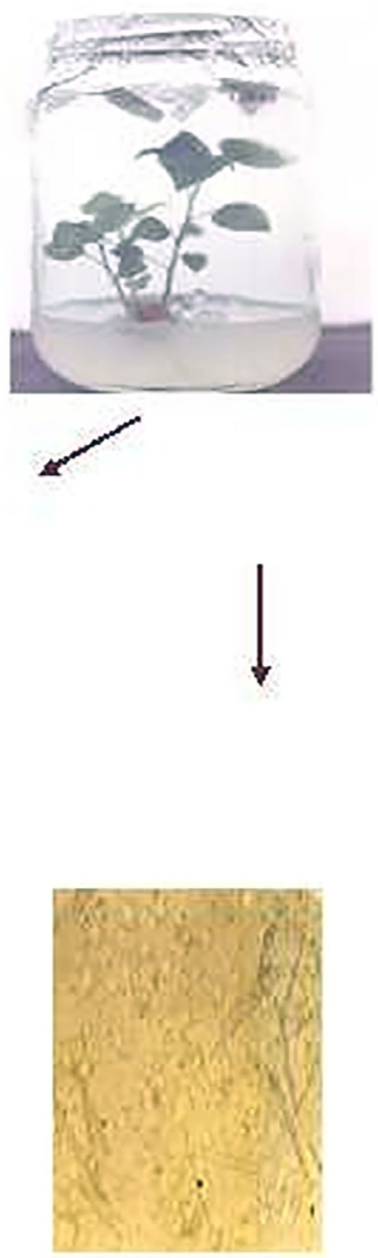
Therapeutic evaluation

Regarding cancer cell cytotoxicity, the results illustrated that in vitro isolated glycoalkaloids, exhibited significant cytotoxic activity against the examined strains from human carcinoma cell lines of lymphoplastic leukemia, lung, liver, and brain (Figure7).

Table 2. Cytotoxicity of in vitro glycoalkaloids from S. nigrum var. judiacium.

\begin{tabular}{lllll}
\hline \multirow{2}{*}{ Cell line } & & & $I_{50}(\mu \mathrm{g} / \mathrm{mL})$ & Tax \\
\cline { 2 - 5 } & Glyc. & Cis & Dox & \\
\hline Lung (H460) & 3.96 & 4.77 & & \\
Brain (U251) & 10.59 & 2.30 & & 0.60 \\
Liver (Hepg2) & 14.1 & 5.99 & & \\
Lymphoblastic leukemia & 18.7 & & & 6.71 \\
Breast (MCF7) & 25.90 & & & \\
\hline Glyc: alycoalkaloids: Cis: cisplatin: Dox: Doxorubicin; Tax: Taxol. & & &
\end{tabular}

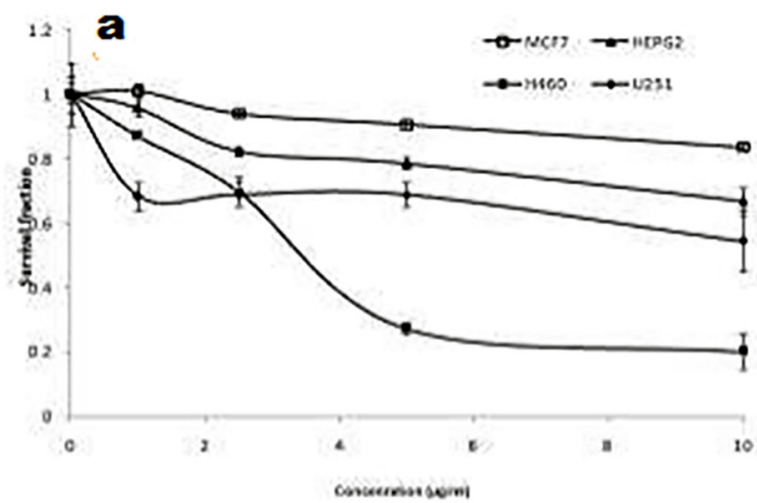

b

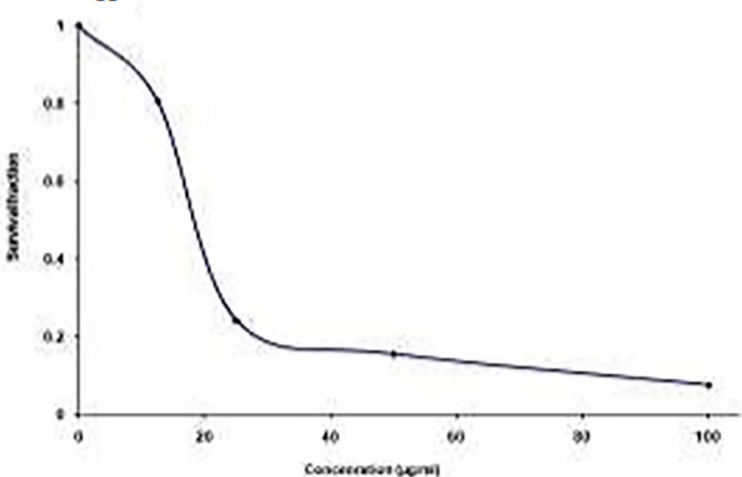

Figure 7. Cytotoxic activity of tissue culture derived glycoalkaloids of S. nigrum var. judiacium against: a- breast, liver, lung and brain carcinoma cell lines; b-lymphoblastic leukemia cell line

$I_{50}$ values comparing with standard cytotoxic drugs are presented (Table 2). The examined cell lines were inhibited in different degrees while control normal cells were unaffected. Lung carcinoma cells were the most susceptible cells to inhibition. Cytotoxic activity might be a virtue to the presence of solasonine, solamargine and solanine. This present outcome is compatible with studies reported that solasonine, B1-solasonine, solamargine and solanigroside $P$ purified from $S$. nigrum have cytotoxicity to
MGC-803 cells and may be potential candidates for the treatment of gastric ulcer (Ding et al., 2013). In the same line, callus methanolic extract of $S$. tuberosum including solanine exhibited pronounced cytotoxicity against different human carcinoma cell lines (Al Ashaal, 2010). Supporting the current data, a-solanine, had shown inhibitory effect on multiple cancer cells, such as clone, liver, melanoma cancer cells in addition to its beneficial effects on pancreatic cancer in vitro and in vivo (Lv et al., 2014). In addition, in vitro 
cytotoxicity of chloroform fruit extract of Solanum nigrum L. against Non Hodgkin Lymphoma (SR) cells was performed and IC50 was $669.27 \mu \mathrm{g} / \mathrm{mL}$ (Huda et al., 2015). Meanwhile, in the present study, purified in vitro glycoalkaloids have potent cytotoxic effect against lymphoma (1301) cell lines with $\mathrm{IC}_{50} 18.7 \mathrm{\mu g} / \mathrm{mL}$.

Concerning with inflammation, the current study showed that, isolated glycoalkaloids displayed outstanding anti-inflammatory activity (Table 3). The activity was 1.2 folds increase than standard indomethacin at lower glycoalkaloids dose $(8 \mathrm{mg} / \mathrm{kg}$ ) comparing with indomethacin (20 mg/kg). At the higher dose $(16 \mathrm{mg} / \mathrm{kg})$ the activity was 1.45 folds that of reference drug indomethacin. Relative to the present data, decoction of the S.nigrum plant depressed the CNS and reflexes of the spinal cord while, the whole plant was used as anti-inflammatory and aqueous plant extracts possessed antiproliferative activity as demonstrated by growth inhibition of cervical carcinoma (Ramalingum\& Mahomoodally, 2014). As well, the anticancer activity of $S$. muricatum fruit extract was associated with immunomodulatory and anti-inflammatory activities (Shathish, \& Guruvayoorappan, 2014).

Table 3. Anti-inflammatory activity of in vitro glycoalkaloids of S. nigrum var. judiacium.

\begin{tabular}{lcrrc}
\hline & \multicolumn{4}{c}{ \%of inhibition } \\
\cline { 2 - 5 } & $1^{\text {st }} \mathrm{h}$ & $2^{\text {nd }} \mathrm{h}$ & $3^{\text {rd }} \mathrm{h}$ & $4^{\text {th }} \mathrm{h}$ \\
\hline Glycoalkaloids $(8 \mathrm{mg} / \mathrm{kg})$ & 36.231 & 93.43 & 90.04 & 94.74 \\
Glycoalkaloids $(16 \mathrm{mg} / \mathrm{kg})$ & 58.23 & 86.85 & 93.54 & 113.85 \\
Indomethacin $(20 \mathrm{mg} / \mathrm{kg})$ & 38.10 & 67.89 & 86.20 & 78.48 \\
\hline
\end{tabular}

Thus the anti-inflammatory activity of the glycoalkaloids in the present study, support the detected cytotoxic activity. Furthermore, the present results could be useful for developing natural treatment for psoriasis whereas, psoriasis is a chronic, proliferative, and inflammatory skin disease with reactive abnormal epidermal differentiation and hyper proliferation that affects 2-3\% of the global population (Garg et al., 2014).

With respect to antioxidant activity, the isolated glycoalkaloids extract manifested emphatic antioxidant capacity (69.98 \%) comparing with standard a-solanine (44\%) and standard vitamin C (100\%). Thus combined glycoalkaloids exerted synergetic antioxidant activity comparing with individual a-solanine. The antioxidant capacity of the isolated glycoalkaloids might be an additional factor explaining their cytotoxic activity against the examined carcinoma cell lines. The key element in linking environmental toxicity to the multistage carcinogenic process is oxidative stress (Tarlovsky, 2013). Besides, S.nigrum herb and its phenolics content were reported to elaborate antioxidant properties (Kumar et al., 2012; Noumedem et al., 2013; Sharma et al., 2014). Based on the present results the glycoalkaloids could act as defensive and protective agent against oxidative hazards.
Due to the scavenging power, glycoalkaloids may neutralize free radicals that cause cell damage and are responsible for oxidative stress and harmful diseases including cancer.

Related to virucidal activity, the safe non toxic concentrations of the glycoalkaloids towards vero cells ranged between 10-50 $\mu \mathrm{g} / \mathrm{mL}$. Table 4 illustrates that purified glycoalkaloids had noticeable inhibitory effect against HSV-1 virus (88.9. \%) at concentration $20 \mu \mathrm{g} / \mathrm{mL}$. Acyclovir at the same concentration exhibited no virucidal activity. At higher concentration $(50 \mu \mathrm{g} / \mathrm{mL}$ ) glycoalkaloids had $94.0 \%$ virucidal inhibitory effect comparing with $89.9 \%$ activity of acyclovir. This result is remarkable due to risky effects of HSV I which is the cause of cornea ulcer and brain damage (Lennerra, 1992). The current antiviral activity of established in vitro glycoalkaloids may be a result of their antioxidant property. In the same line, the antiviral activity of Morus alba extract against Herpes simplex virus type 1 and 2 was returned to its antioxidant activity (Dkhil, 2015). The present results are of special interest for developing new natural antiviral treatment from S.nigrum var. judiacium. Herpes simplex viruses 1 and 2 are the cause of dangerous diseases as oral herpes and genital lesions. Infection of HSV is the most common causes of meningitis and 
encephalitis when it infects the central nervous system (Dkhil, 2015). HSV infection may be lethal in immunocompromised patients. Globally, $45 \%$ to $98 \%$ of the world population are infected or previously infected with Herpes simplex virus type 1 (Dkhil, 2015). Furthermore relative high side effects and emergence of drug- resistant virus strains are major complications associated with treatment of Herpes infections.

Table 4. Virucidal effect of in vitro glycoalkaloids of S. nigrum var. judiacium. against HSV-1.

\begin{tabular}{cccc}
\hline & \multicolumn{3}{c}{ HSV-1 } \\
\cline { 2 - 4 } & Initial count (PFU/mL) & Treated count (PFU/mL) & \% of virus reduction \\
\hline Glyc. $20 \mu \mathrm{g}$ & $9.76 \times 10^{4}$ & $1.08 \times 10^{4}$ & $88.9 \%$ \\
Acy. $20 \mu \mathrm{gg}$ & $9.76 \times 10^{4}$ & $9.76 \times 10^{4}$ & $0 \%$ \\
Glyc. $50 \mu \mathrm{g}$ & $9.76 \times 10^{4}$ & $0.58 \times 10^{4}$ & $94.0 \%$ \\
Acy. $50 \mu \mathrm{gg}$ & $9.76 \times 10^{4}$ & $1.08 \times 10^{4}$ & $88.9 \%$ \\
N.C & $9.76 \times 10^{4}$ & $9.76 \times 10^{4}$ & - \\
\hline PFU/mL; plaque forming unit/mL. HSV-1; Herpes simplex virus type 1. Glyc..; Glycoalkaloids from of S. nigrum var. judiacium callus cultures. Acy. ; acyclovir. N.C.: \\
negative control.
\end{tabular}

As Regard to hepatic vermfuge assay, the glycoalkaloids showed schistomicidal activity against S. mansoni with $\mathrm{LC}_{50}$ values $132.8,83.9,76.4$ and 69.3ppm. While LC $_{90}$ values were 179.7, 120.1, 104.9 and 89.5 ppm, respectively after 48,72 , and 96 and $120 \mathrm{~h}$. Concerning fasciolicidal effect of the glycoalkaloids, $\mathrm{LC}_{50}$ and $\mathrm{LC}_{90}$ were 76.60 and 114.41 ppm, respectively after $24 \mathrm{~h}$. Braziquantel as positive control induced $100 \%$ mortality after $24 \mathrm{~h}$ at 100 ppm for both worms, while negative control groups remained survival all over the experiment period. Analysis was performed using probit program and $\mathrm{LC}_{50}$ determined at 95\% confidence limit. The results of this part of study could be considered as a promising strategy to control the disease and are in close agreements studies which reported that dry powdered S. nigrum leaf exhibited potent effect in disturbing the intermediate host for S. mansoni (Biomphalaria snail) biochemistry which may render them physiologically unsuitable for the developing of schistosome parasite (Al-Daihan, 2008). Antifasciolosis effect of pure glycoalkaloids in the current research is in consistence with the reported potent molluscicidal activity of hydro methanol immature fruit extract of Solanum nigrum var. villosum against Fasciola hepatica intermediate host Galba truncatula (Hammami et al., 2011). The current results may be a guide for elaborating anti parasitic remedies where fasciolosis and schistomiasis are common diseases.

\section{Conclusion}

The present outcomes ultimately encourage glycoalkaloids production from Solanum nigrum var. judiacium via tissue culture way. The increments ranged from 2.51 to 5.17 folds that of the parent plant. This overflow production allows amalgamation of these potent metabolites from botanical origin in pharmaceutical industry. The derived glycoalkaloids possessed antiinflammatory activity represents 1.2- 1.5 folds that of indomethacin. The most affected cell line is that of lung ( $I C_{50}$ is 0.83 that of cisplatin). The produced glycoalkaloids also performed antiparasitic effect against liver parasites ( $S$. mansoni and F. gigantica). The confirmed positive biological results illustrate substantially possible future formulation of these high potency glycoalkaloids as prodrugs from botanical origin that serve in treatments of critical diseases and could help to overcome the problems of resistance to drugs currently in use.

\section{Conflict of interest}

The author has no conflict of interest.

\section{Acknowledgment}

Deep thanks to National Research Centre for funding this research. The author also wishes to thank NRC, National Cancer Institute and Theodor Belharz Institute, Egypt for making their facilities available.

\section{References}

Al- Daihan, S. 2008. Measurement of selected enzymatic activities in Solanum nigrum-treated Biomphalaria arabica Snails. Journal of Applied Sciences 8: 881-885.

Al-Ashaal, H. A. 2010. Regeneration, in vitro 
glycoalkaloids production and evaluation of bioactivity of callus methanolic extract of Solanum tuberosum L. Fitoterapia 81: 600-606.

Al-Ashaal, H.A., Aboutabl, M.E., Maklad, Y.A., El-Beih, A.A. 2013. Tropane alkaloids of Atropa belladonna L.: in-vitro production and pharmacological profile. Egyptian Pharmaceutical Journal 12: 130-135.

Bhatnagar, P., Bhatnagar, M., Nath, A. K., Sharma, D.R. 2004. Production of solasodine by Solanum laciniatum using plant tissue culture technique. Indian Journal of Experimental Biollogy 42: 10201023.

Ding, X., Zhu, F., Yang, Y., Li, M. 2013. Purification, antitumor activity in vitro of steroidal glycoalkaloids from black nightshade (Solanum nigrum L.). Journal of Food Chemistry 141: 11811186.

Dkhil, M., Quraishy, S., Delic, D. 2015. The antioxidant and anti-Herpes simplex viruses activity of Morus alba leaves extract. Pakistan Journal of Zoology 47: 1563-1569.

El-Ashaal, H.A., Ghanem, S.A., Melek, F.R., Kohail, M.A., Hilal, S.H. 1999. Alkaloid production from regenerated Solanum plants. Fitoterapia 70: 407411.

Finney, D.J. 1971. Probbit Analysis. Cambridge University Press, Cambridge. 333 p

Garg, T., Rath, G., Goyal, A.K., 2014. Ancient and advanced approaches for the treatment of an inflammatory autoimmune disease-psoriasis. Critical Reviews in Therapeutic Drug Carrier Systems 31: 331-364.

Gheewalaa, N., Saralayab, M., Sonarac, G., Gheewalaa, T. 2013. Phytochemical Evaluation of total glycoalkaloid of dried fruit of Solanum nigrum Linn. Current Pharma Research 3: 10101013.

Hammami, H., Mezghani-Jarraya, R. M., Damak, M., Ayadi, A. 2011. Molluscicidal activity of various solvent extracts from Solanum nigrum var. villosum L. aerial parts against Galba truncatula. Parasite 18: 63-70.

Hansen, M.B., Nielsen, S.E., Berg, K. 1989. Reexamination and further development of a precise and rapid dye method for measuring cell growth/cell kill. Journal of Immunological Methods 119: 203-210.

Hassanain, M.A., Abdel Rahman, E.H., Abdel Megeed, K.A.N. 1998. Vermicidal effect of Bacillus thuringiensis on Fasciola gigantica. Journal of Egyptian Veterinary Medical Association 1 58: 251- 267.
Huda K. A., Saadi, K., Nahi, Y. 2015. Evaluation of the activity of the chloroform extract of Solanum nigrum on non Hodgkin lymphoma cell line (REF) and human lymphocytes in vitro. World $J$ of Pharmacy and Pharmaceutical Sciences 4: 1422.

Kumar, A., Sagwal, S., Niketa, Rani, S. 2012. An updated review on molecular genetics, phytochemistry, pharmacology and physiology of black night shade (Solanum nigrum). International Journal of Pharmaceutical Sciences and Research 3: 2955-2977.

Lennette E.H.1992. Laboratory Diagnosis of Viral Infections. Dekker press, NewYork, Marcel. 351 p.

Lindesy, K., Yeoman, M. 1983. The Relationship between growth rate, differentiation and alkaloid accumulation in cell cultures. Journal of Experimental Botany 34, 1055-1065.

Lv, C., Kong, H., Dong, G. , Liu, L., Tong, K., Sun, H., Chen, B., Zhang, C., Zhou M. 2014. Antitumor efficacy of a-solanine against pancreatic cancer in Vitro and in Vivo. PLOS one. Available at http:// dx.doi.org/10.1371/journal.pone. 0087868.

Misawa, M., 1994. Plant Tissue Culture: An Alternative for Production of Useful Metabolites, Food and Agriculture Organization of The United Nations, Rome, FAO, Bulletin No 108, International Inc.Toronto, Canada. 87 p.

Moreeira, C.B.1., Lima, S.S.1., Esquibele, M.A.1., Sato, A.1. 2010. Solasodine accumulation in regenerated plants of Solanum torvum SW. A Revista Brasileira de Plantas Medicinais 12: 73-79.

Murashige, T., Skoog, F., 1962. A revised medium for rapid growth and bioassays with tobacco tissue cultures. Physiologia Plantarum 15: 973-997.

Noumedem, J., Mihasan, M., Lacmata, S., Stefan, M., Kuiate, J., Kuete, V. 2013. Antibacterial activities of the methanol extracts of ten Cameroonian vegetables against Gramnegative multidrug-resistant bacteria. BMC Complementary and Alternative Medicine 31: 13-26.

Papageorgiou, G., Laura, L., Christodoulou, C.G., Lucena, F., Akkelidou, D., Loannou, E., Jofre, J. 2000. A simple methodological approach for counting and identifying cytopathogenic viruses adsorbed to cellulose nitrate membrane filters. Applied and environmental microbiology 66: 194- 198.

Ramalingum, N., Mahomoodally, F. 2014. The therapeutic potential of medicinal foods. Advances in pharmacological sciences 2014: Article ID 354264, 18 pages. Available at http:// dx.doi.org/10.1155/2014/354264 
Sharma, M., Romani, M., Menaria, J., Devi, S., Sheikh, M. 2014. In vitro antioxidant potential of various extracts of Solanum nigrum $L$. The Pharmaceutical and Chemical Journal 1: 6-9.

Shathish, K., Guruvayoorappan, C. 2014. Solanum muricatum Ait. inhibits inflammation and cancer by modulating the immune system. Journal of Cancer Research and Therapeutics 10: 623- 630.

Skehan, P., Storeng, R., Scudiero, D., Monks, A., McMahon J, Vistica, D., Warren, J., Bokesch, H., Kenney, S., Boyd, M. 1990. New colorimetric cytotoxic assay for anticancer-drug screening. Journal of National Cancer Institute 82: $1107-$ 1112.

Tarlovsky, V. 2013. Role of antioxidants in cancer therapy, Nutrition 29: 15-21.

Tek, N. 2006. Chromatographic determination of glycoalkaloids in egg plant. Master Thesis., Izmir Institute of Technology, Izmir, Turkey, 86 p.

Tiossi, R., Miranda, M., Sousa, J., Prac, F., Bentley, M., Chesney, J., Bastos, J. 2012. A Validated reverse phase HPLC analytical method for quantitation of glycoalkaloids in Solanum lycocarpum and its extracts. Journal of Analytical Methods in Chemistry 2012: Article ID 947836, 8 pages doi:10.1155/2012/947836.

Väänäne, T. 2007. Glycoalkaloid content and starch structure in Solanum species and interspecific somatic potato hybrids. Ph.D. Thesis, University of Helsinki, Helsinki, 79 p.

Williams, C. 2012. Medicinal Plants in Australia: Plants, Potions and Poisons. Rosenberg, Australia, $472 \mathrm{p}$.

Winter, G.A., Risley, E.A., Nuss, G.W. 1962. Carrageenan-induced oedema in hind paw of the rat as an assay for anti-inflammatory drugs. In: Proceedings of the Society for Experimental Biology and Medicine, III, 1544-1547.

Xu, K., Chang, Y., Liu, K., Wang, F., Liu, Z., Zhang, T., Li, T., Zhang,Y., Zhang, F, Wang,Y., Niu, W., Jia, S., Xie, H., Tan, G., Li, C. 2014. Regeneration of Solanum nigrum by somatic embryogenesis, involving frog egg-like body, a novel structure. PLoS One 4:9(6):e986724. 